quant-ph/9804056

CBPF-NF/007/98

April 22, 1998

Revised June 2, 1998

\title{
Vacuum Energy Density near Fluctuating Boundaries
}

\author{
L.H. Ford円 \\ Institute of Cosmology, Department of Physics and Astronomy \\ Tufts University \\ Medford, Massachusetts 02155 \\ N.F. Svaiter \\ Centro Brasileiro de Pesquisas Fisicas-CBPF \\ Rua Dr. Xavier Sigaud 150 \\ Rio de Janeiro, RJ 22290-180, Brazil
}

\begin{abstract}
The imposition of boundary conditions upon a quantized field can lead to singular energy densities on the boundary. We treat the boundaries as quantum mechanical objects with a nonzero position uncertainty, and show that the singular energy density is removed. This treatment also resolves a long standing paradox concerning the total energy of the minimally coupled and conformally coupled scalar fields.
\end{abstract}

PACS categories: 03.70.+k, 12.20.Ds, 04.62.+v.

\footnotetext{
${ }^{1}$ email: ford@cosmos2.phy.tufts.edu

${ }^{2}$ email:nfuxsvai@lafex.cbpf.br
} 


\section{Introduction}

It is well-known that boundary conditions imposed upon quantum fields may lead to divergent expectation values for local observables. A simple example is a massless, minimally coupled scalar field $\varphi(t, \mathbf{x})$ which vanishes on the $z=0$ plane;

$$
\left.\varphi\right|_{z=0}=0 .
$$

One finds [1] that the renormalized expectation values of both $\varphi^{2}$ and of the energy density $T_{t t}$ diverge as $z \rightarrow 0$. Specifically,

$$
\left\langle\varphi^{2}\right\rangle=-\frac{1}{16 \pi^{2} z^{2}}
$$

and

$$
\left\langle T_{t t}\right\rangle=-\frac{1}{16 \pi^{2} z^{4}} .
$$

(Units in which $\hbar=c=1$ will be used throughout this paper. The metric tensor is taken to be $\eta_{\mu \nu}=\operatorname{diag}(1,-1,-1,-1)$.) The stress energy tensor of the massless minimally coupled scalar field is given by

$$
T_{\mu \nu}=\partial_{\mu} \varphi \partial_{\nu} \varphi-\frac{1}{2} \eta_{\mu \nu} \partial^{\alpha} \varphi \partial_{\alpha} \varphi
$$

Similar divergences occur in the expectation values of the squared electric or magnetic fields, $\left\langle\mathbf{E}^{2}\right\rangle$ or $\left\langle\mathbf{B}^{2}\right\rangle$ near a perfectly conducting plane, although in this case the local energy density remains finite. When the conducting boundary is curved, then the energy density diverges on the boundary [2].

Furthermore, there is a puzzling discrepancy between the Casimir energy for a minimal scalar field computed as the renormalized expectation value of the Hamiltonian and as a spatial integral of $\left\langle T_{t t}\right\rangle$. Consider the case of two parallel plates with separation $L$ on which the field vanishes. If we first form the Hamiltonian operator $H=\int T_{t t} d^{3} x$, the result is the same for both the minimal and conformally coupled fields. This follows from the fact that the stress tensor for the conformal field,

$$
\Theta_{\mu \nu}=\partial_{\mu} \varphi \partial_{\nu} \varphi-\frac{1}{2} \eta_{\mu \nu} \partial^{\alpha} \varphi \partial_{\alpha} \varphi-\frac{1}{6}\left[\partial_{\mu}\left(\varphi \partial_{\nu} \varphi\right)+\partial_{\nu}\left(\varphi \partial_{\mu} \varphi\right)-2 \eta_{\mu \nu} \partial^{\alpha}\left(\varphi \partial_{\alpha} \varphi\right)\right]
$$

differs from that for the minimal field, Eq. (1), by a total derivative term which integrates to zero. If we find the renormalized expectation value of $H$, the energy per unit area is found to be $-\pi^{2} /\left(1440 L^{3}\right)$. However, if we attempt to compute this energy per unit area as $\int_{0}^{L}\left\langle T_{t t}\right\rangle d z$, the result is divergent. This discrepancy has led some authors [3] to postulate the existence of a singular surface energy density, which would render the latter expression finite and equal to the former result. (Note that the surface energy densities which are of concern here are distinct from the surfacearea-dependent terms in the regularized Casimir energy which can arise in particular regularization methods [4, 5].) 
In curved or topologically nontrivial spacetimes, it is also possible for the renormalized expectation value of the stress tensor to diverge on particular boundaries. An example is the Boulware vacuum state in Schwarzschild spacetime, for which the stress tensor diverges on the event horizon [6, 7]. This divergence is usually interpreted as indicating that this is not a physically realizable state. Other examples of divergent stress tensors include Misner space, where the divergence occurs on the Cauchy horizon [8]. In this and similar examples, one is tempted to resolve the problem by regarding the spacetime itself to be unphysical. Indeed, this philosophy is the basis of Hawking's Chronology Protection Conjecture [10], which argues that closed timelike curves are prohibited by the effects of divergent energy densities which would otherwise appear on the chronology horizon (the boundary between a region containing closed timelike curves and one without such curves).

One may understand why the imposition of a boundary condition such as Eq. (1) on a quantum field can result in infinities. In the case of $\left\langle\varphi^{2}\right\rangle$, renormalization means taking the difference of the expectation value in the presence of the boundary and in its absence. Normally, this removes the infinite part and leaves a finite remainder. However, on the boundary the formal expectation value of $\left\langle\varphi^{2}\right\rangle$ is finite, so the subtraction results in an infinite difference. We can also understand why quantities such as $\left\langle\dot{\varphi}^{2}\right\rangle$ and $\left\langle T_{t t}\right\rangle$ become infinite on the boundary. The field $\varphi$ and its time derivative $\dot{\varphi}$ are conjugate variables which satisfy an uncertainty relation. If $\varphi$ is precisely specified, $\dot{\varphi}$ is completely indeterminate, and $\left\langle\dot{\varphi}^{2}\right\rangle$ and thus $\left\langle T_{t t}\right\rangle=\frac{1}{2}\left\langle\dot{\varphi}^{2}+(\nabla \varphi)^{2}\right\rangle$ are infinite. A state in which $\varphi$ is precisely determined at a point has infinite energy density at that point for essentially the same reason that a position eigenstate in single particle quantum mechanics has infinite energy.

In the case of material boundaries, such metal plates, infinite values of $\left\langle\mathbf{E}^{2}\right\rangle$ or other observables are presumably avoided because such boundaries are not perfect reflectors at all frequencies. A metal plate is a good reflector of electromagnetic waves at frequencies below the plasma frequency, but becomes relatively transparent at higher frequencies. Such a high frequency cutoff seems not to be available when the "boundary" is a feature of the spacetime structure.

The purpose of the present paper is to explore an alternative mechanism for introducing a cutoff which removes singular behavior on boundaries. This is to allow the position of the boundary to undergo quantum fluctuations. One might expect that such fluctuations will smear out the contributions of the high frequency modes without the need to introduce an explicit high frequency cutoff.

\section{$2\left\langle\varphi^{2}\right\rangle$ near a Single Plate}

Let us consider a plane boundary located at $z=q$. If we impose the boundary condition on a massless quantized scalar field $\varphi$ that it vanish on this boundary, the appropriate two-point function may be constructed as an image sum. The result is

$$
\left\langle\varphi(x) \varphi\left(x^{\prime}\right)\right\rangle=G\left(x, x^{\prime}\right)=G_{0}\left(x, x^{\prime}\right)+G_{R}\left(x, x^{\prime}\right),
$$


where

$$
G_{0}\left(x, x^{\prime}\right)=-\frac{1}{4 \pi^{2}\left(\Delta t^{2}-\Delta \mathbf{x}^{2}\right)}
$$

is the empty space two-point function, with $\Delta t=t-t^{\prime}, \Delta \mathbf{x}^{2}=\left|\mathbf{x}-\mathbf{x}^{\prime}\right|^{2}$, and

$$
G_{R}\left(x, x^{\prime}\right)=\frac{1}{4 \pi^{2}\left[\Delta t^{2}-\Delta x^{2}-\Delta y^{2}-\left(z+z^{\prime}-2 q\right)^{2}\right]} .
$$

The full two-point function, $G\left(x, x^{\prime}\right)$, vanishes whenever $z=q$ or $z^{\prime}=q$. The renormalized expectation value of $\varphi^{2}$ is given by the coincidence limit of the renormalized two-point function, $G_{R}\left(x, x^{\prime}\right)$,

$$
\left\langle\varphi^{2}\right\rangle=G_{R}(x, x)=-\frac{1}{16 \pi^{2}(z-q)^{2}}
$$

and is singular at $z=q$.

We now wish to allow the position variable $q$ to fluctuate. This will occur if the mirror is treated as a quantum object with a wavefunction $\psi(q)$, and hence a position probability distribution of

$$
f(q)=|\psi(q)|^{2}
$$

where

$$
\int_{-\infty}^{\infty} f(q) d q=1
$$

The average over position of a function $H(q)$ becomes

$$
\langle H\rangle=\int_{-\infty}^{\infty} H(q) f(q) d q
$$

Thus to find the mean value of $\varphi^{2}$, we need to calculate $\left\langle G_{R}\right\rangle$. This is most easily done by expressing $G_{R}$ in a Fourier representation, and then averaging the $q$-dependence of the mode functions:

$$
\left\langle G_{R}\left(x, x^{\prime}\right)\right\rangle=-\frac{1}{2(2 \pi)^{3}} \operatorname{Re} \int \frac{d^{3} k}{\omega} e^{i \mathbf{k}_{t} \cdot\left(\mathbf{x}_{t}-\mathbf{x}_{t}^{\prime}\right)} e^{-i \omega\left(t-t^{\prime}\right)} e^{i k_{z}\left(z+z^{\prime}\right)}\left\langle e^{-2 i k_{z} q}\right\rangle,
$$

where $\mathbf{k}_{t}$ and $\mathbf{x}_{t}$ denote the components of $\mathbf{k}$ and $\mathbf{x}$, respectively, in directions parallel to the plate.

To proceed further, we must specify the probability distribution, $f(q)$. A convenient choice is a Gaussian peaked about $q=0$,

$$
f(q)=\sqrt{\frac{\alpha}{\pi}} e^{-\alpha q^{2}}
$$

which leads to

$$
\left\langle e^{-2 i k_{z} q}\right\rangle=e^{-2 k_{z}^{2}\left\langle q^{2}\right\rangle}
$$


with

$$
\left\langle q^{2}\right\rangle=\frac{1}{2 \alpha} .
$$

This probability distribution is the appropriate one to describe, for example, a plate in the ground state of a harmonic potential. Note that Eq. (15) is equivalent to the result which one obtains when taking the vacuum expectation value of the complex exponential of a free quantum field. (See, for example, Eq. (8) of Ref. [9].) This is to be expected, as a free quantum field in the vacuum state is equivalent to an infinite collection of harmonic oscillators in their ground states.

If we use Eq. (15) in Eq. (13), set $\mathbf{x}^{\prime}=\mathbf{x}$, and use

$$
\int d^{3} k=2 \pi \int_{-\infty}^{\infty} d k_{z} \int_{\left|k_{z}\right|}^{\infty} d \omega \omega
$$

for integrands independent of the azimuthal angle, the result is

$$
\left\langle G_{R}\left(t, t^{\prime}\right)\right\rangle=\frac{1}{2(2 \pi)^{2}\left(t-t^{\prime}\right)} \operatorname{Re}\left[i \int_{-\infty}^{\infty} d k_{z} e^{i\left(2 z k_{z}+\left(t-t^{\prime}\right)\left|k_{z}\right|\right)} e^{-2 k_{z}^{2} \Delta^{2}}\right]
$$

where

$$
\Delta=\sqrt{\left\langle q^{2}\right\rangle}
$$

is the root-mean-squared displacement of the mirror. This integral may be performed in terms of the error function $\Phi$ to be

$$
\begin{aligned}
\left\langle G_{R}\left(t, t^{\prime}\right)\right\rangle & =-\frac{i \sqrt{2 \pi}}{8(2 \pi)^{2}\left(t-t^{\prime}\right) \Delta}\left[e^{-\left(2 z-t+t^{\prime}\right)^{2} /\left(8 \Delta^{2}\right)} \Phi\left(i \frac{t-t^{\prime}-2 z}{2 \sqrt{2} \Delta}\right)\right. \\
& \left.+e^{-\left(2 z+t-t^{\prime}\right)^{2} /\left(8 \Delta^{2}\right)} \Phi\left(i \frac{t-t^{\prime}+2 z}{2 \sqrt{2} \Delta}\right)\right] .
\end{aligned}
$$

(Here and at many other places in this paper, the calculations were performed with the aid of the symbolic algebra program MACSYMA.) This quantity is finite in the limit that $t^{\prime} \rightarrow t:$

$$
\left\langle G_{R}\right\rangle=\frac{\sqrt{2} z}{32 \sqrt{\pi^{3}} \Delta^{3}} e^{-z^{2} /\left(2 \Delta^{2}\right)} i \Phi\left(i \frac{z}{\sqrt{2} \Delta}\right)+\frac{1}{16 \pi^{2} \Delta^{2}} .
$$

Note that this expression is real, as may be seen from the fact that

$$
\Phi(i x)=-\Phi(-i x)=\frac{2 i}{\sqrt{\pi}} \int_{0}^{x} e^{u^{2}} d u .
$$

The quantity $\left\langle G_{R}\right\rangle$ is $\left\langle\varphi^{2}\right\rangle$ in the presence of position fluctuations, and is finite for all $z$. For large $z$, we have

$$
\left\langle G_{R}\right\rangle \sim-\frac{1}{16 \pi^{2} z^{2}}-\frac{3 \Delta^{2}}{16 \pi^{2} z^{4}}+\cdots, \quad z \gg \Delta,
$$




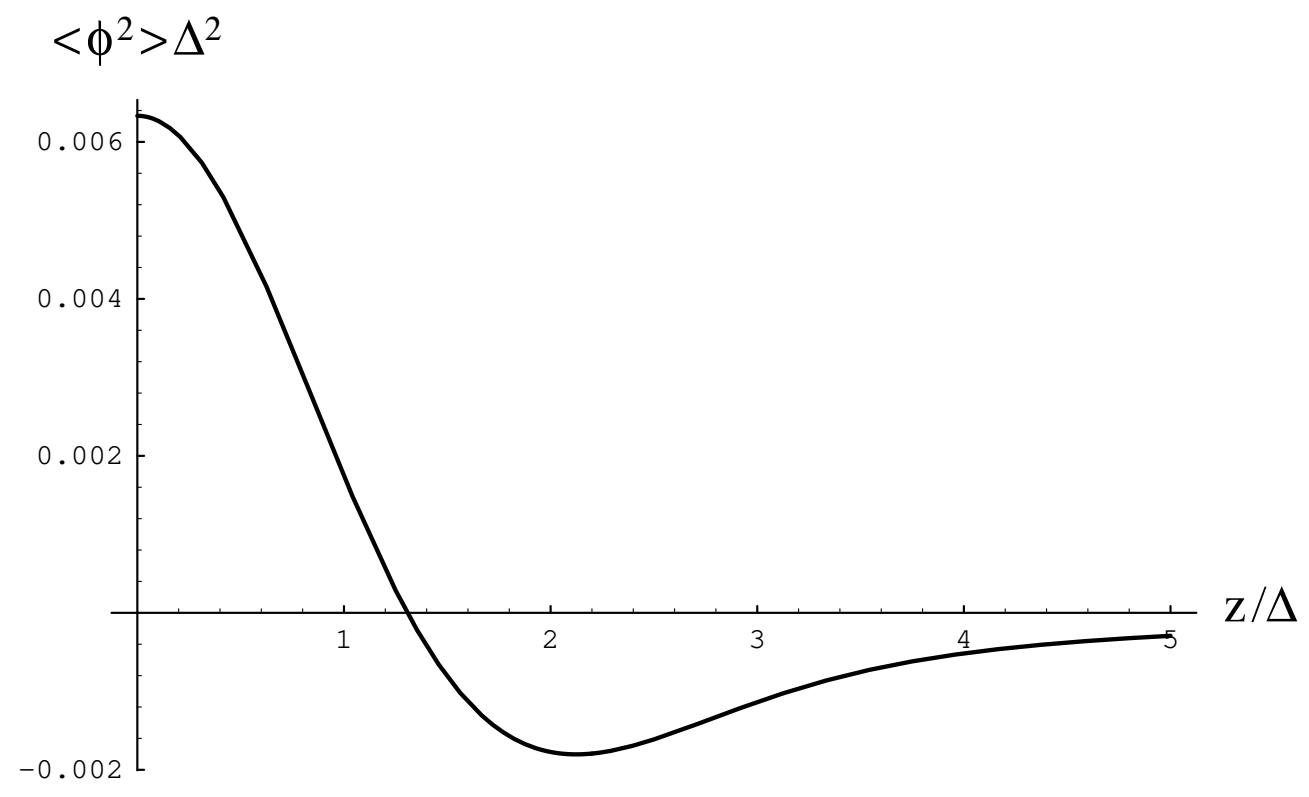

Figure 1:

The mean value of $\varphi^{2}$ near a mirror undergoing Gaussian position fluctuations is shown. Here $\Delta$ is the characteristic width of the probability distribution. For $z / \Delta$ large, $\left\langle\varphi^{2}\right\rangle$ is approximately given by Eq. (2), but it is finite as $z / \Delta \rightarrow 0$.

thus recovering the usual form far from the mirror. As $z \rightarrow 0$,

$$
\left\langle G_{R}\right\rangle \rightarrow \frac{1}{16 \pi^{2} \Delta^{2}},
$$

and is hence finite. For intermediate values of $z,\left\langle G_{R}\right\rangle$ may be computed numerically, and is depicted in Fig. 1.

\section{The Energy Density near a Single Plate}

\subsection{Gaussian Fluctuations}

We now wish to apply the procedure used in the previous section to find the energy density in the presence of a single plate, whose position is undergoing fluctuations with a Gaussian probability distribution. This energy density may be expressed as

$$
\langle\rho\rangle=\frac{1}{2} \lim _{\substack{t^{\prime} \rightarrow t \\ \mathbf{x}^{\prime} \rightarrow \mathbf{x}}}\left(\partial_{t} \partial_{t}^{\prime}+\nabla_{\mathbf{x}} \cdot \nabla_{\mathbf{x}^{\prime}}\right)\left\langle G_{R}\left(x, x^{\prime}\right)\right\rangle .
$$


A repetition of the procedure used for $\left\langle G_{R}\right\rangle$ leads to

$$
\langle\rho\rangle=-\frac{1}{2(2 \pi)^{3}} \lim _{\substack{t^{\prime} \rightarrow t \\ \mathbf{x}^{\prime} \rightarrow \mathbf{x}}} \operatorname{Re} \int \frac{d^{3} k}{\omega}\left(\omega^{2}-k_{z}^{2}\right) e^{i \mathbf{k}_{t} \cdot\left(\mathbf{x}_{t}-\mathbf{x}_{t}^{\prime}\right)} e^{-i \omega\left(t-t^{\prime}\right)} e^{i k_{z}\left(z+z^{\prime}\right)}\left\langle e^{-2 i k_{z} q}\right\rangle .
$$

If we now employ the relation for Gaussian fluctuations, Eq. (15), and perform the integrations as before, we find

$$
\begin{aligned}
\langle\rho\rangle & =\frac{2 i}{(2 \pi)^{3} \Delta} \sqrt{\frac{\pi}{2}} \lim _{t^{\prime} \rightarrow t} \frac{\partial^{2}}{\partial u \partial v}\left\{\frac { 1 } { u + v } \left[e^{-u^{2} /\left(8 \Delta^{2}\right)} \Phi\left(i \frac{u}{2 \sqrt{2} \Delta}\right)\right.\right. \\
& \left.\left.+e^{-v^{2} /\left(8 \Delta^{2}\right)} \Phi\left(i \frac{v}{2 \sqrt{2} \Delta}\right)\right]\right\}
\end{aligned}
$$

where $u=t-t^{\prime}-2 z$, and $v=t-t^{\prime}+2 z$. Explicit evaluation of the last expression leads to

$$
\langle\rho\rangle=\frac{1}{192 \pi^{2} \Delta^{7}}\left[\sqrt{2 \pi} z\left(z^{2}-3 \Delta^{2}\right) e^{-z^{2} /\left(2 \Delta^{2}\right)} i \Phi\left(i \frac{z}{\sqrt{2} \Delta}\right)+2 \Delta\left(z^{2}-2 \Delta^{2}\right)\right] .
$$

Far from the mirror, the energy density is that calculated without fluctuations:

$$
\langle\rho\rangle \sim-\frac{1}{16 \pi^{2} z^{4}}+\cdots, \quad z \gg \Delta,
$$

and near the mirror it is finite

$$
\langle\rho\rangle \rightarrow-\frac{1}{48 \pi^{2} \Delta^{4}} \quad z \rightarrow 0 .
$$

The energy density, $\langle\rho\rangle$ is given as a function of $z$ in Fig. 2.

The remaining components of the expectation value of the stress tensor, $\left\langle T_{\mu \nu}\right\rangle$, may be readily obtained. This must be a Lorentz tensor formed from the metric $\eta_{\mu \nu}$ and $n_{\mu} n_{\nu}$, where $n^{\mu}=(0,0,0,1)$ is the unit normal vector to the mirror. Hence,

$$
\left\langle T_{\mu \nu}\right\rangle=F_{1}(z) \eta_{\mu \nu}+F_{2}(z) n_{\mu} n_{\nu}
$$

where $F_{1}$ and $F_{2}$ are scalar functions of $z$. We see immediately that the transverse components are just minus the energy density:

$$
\left\langle T_{x x}\right\rangle=\left\langle T_{y y}\right\rangle=-\langle\rho\rangle .
$$

Furthermore, the conservation law, $\partial^{\mu}\left\langle T_{\mu \nu}\right\rangle=0$ implies that

$$
\frac{d}{d z}\left[F_{1}(z)-F_{2}(z)\right]=0 .
$$

We define the renormalized stress tensor so that $\left\langle T_{\mu \nu}\right\rangle \rightarrow 0$ as $z \rightarrow \infty$, which implies $F_{1}(z)=F_{2}(z)$, and hence

$$
\left\langle T_{\mu \nu}\right\rangle=\langle\rho\rangle\left(\eta_{\mu \nu}+n_{\mu} n_{\nu}\right)
$$




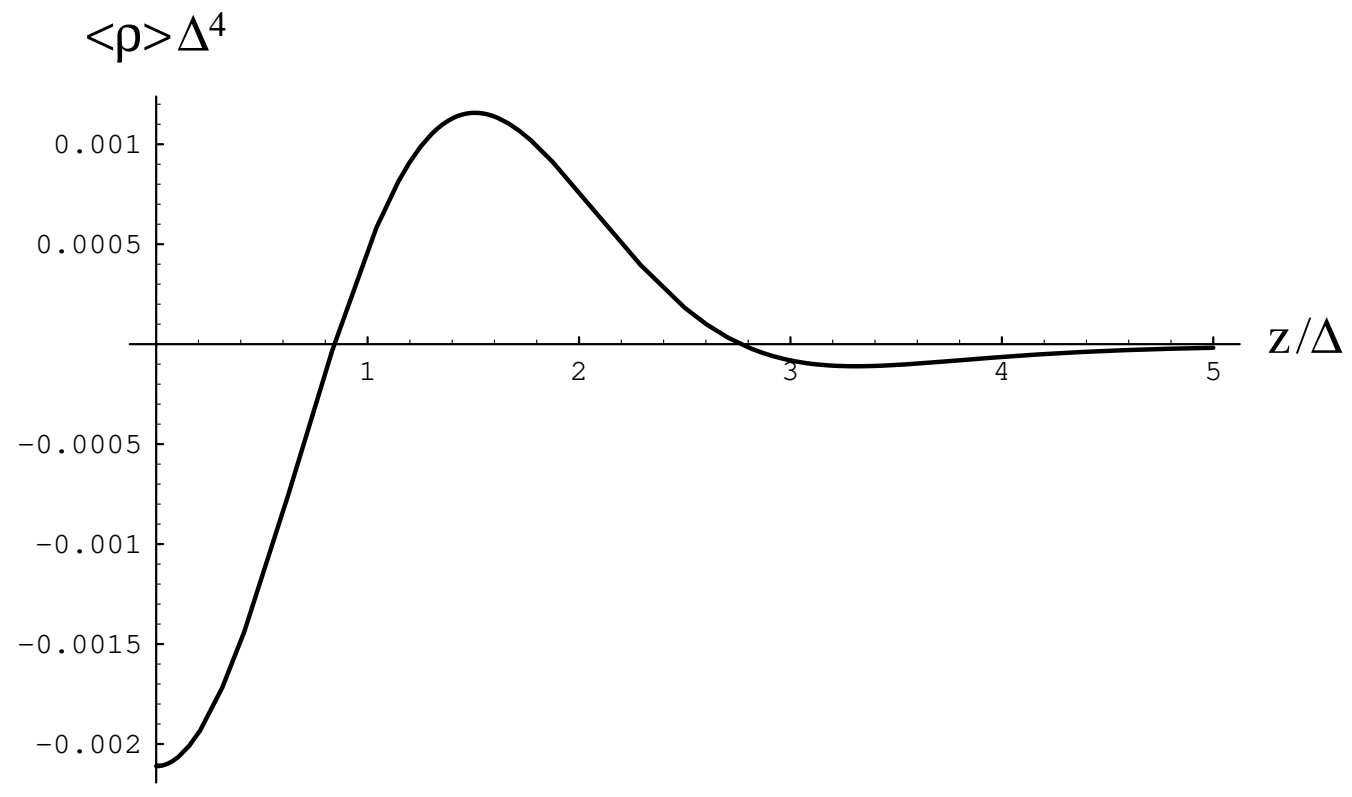

Figure 2:

The mean energy density, $\langle\rho\rangle$, near a mirror undergoing Gaussian position fluctuations is shown. The integral of this function over all positive $z$ vanishes.

As a consequence, the pressure normal to the mirror vanishes:

$$
\left\langle T_{z z}\right\rangle=0 .
$$

Finally, we note that one may calculate the integral of the right-hand-side of Eq. (28) explicitly and verify that

$$
\int_{0}^{\infty}\langle\rho\rangle d z=0 .
$$

This confirms that the boundary fluctuations remove the apparent discrepancy between the Casimir energies of the minimal and conformal scalar fields. Note that although $\langle\rho\rangle$ is negative both at large distances and at the mean position of the mirror, it is positive in a region near $z=0$, as illustrated in Fig. 2. The positive energy region can be regarded as the concrete realization of the positive surface energy density conjectured by Kennedy, et al [3].

\subsection{A General Probability Distribution}

Now we wish to generalize our discussion to an arbitrary probability distribution. For later use, we will momentarily assume that the distribution function $f$ is peaked 
symmetrically around an arbitrary value of $\langle q\rangle$, and write $f=f(s)$, where $s=q-\langle q\rangle$. The average of a complex exponential function of $q$ then becomes

$$
\left\langle e^{i \alpha q}\right\rangle=e^{i \alpha\langle q\rangle} \hat{f}(\alpha),
$$

where $\hat{f}$ denotes the Fourier transform of $f$ :

$$
\hat{f}(\alpha)=\int_{-\infty}^{\infty} e^{i \alpha s} f(s) d s
$$

We may now use Eq. (26) to express the averaged energy density for an arbitrary, symmetric probability distribution as

$$
\langle\rho\rangle=-\frac{1}{2 \pi^{2}} \lim _{t^{\prime} \rightarrow t} \frac{\partial^{2}}{\partial u \partial v} \operatorname{Re}\left\{\frac{i}{u+v} \int_{0}^{\infty} d k_{z}\left[e^{-i k_{z} u} \hat{f}\left(-2 k_{z}\right)+e^{-i k_{z} v} \hat{f}\left(2 k_{z}\right)\right]\right\},
$$

where, as before, $u=t-t^{\prime}-2 z$, and $v=t-t^{\prime}+2 z$. We next use Eq. (38) to re-express $\langle\rho\rangle$ in terms of $f$, and employ the relation

$$
\int_{0}^{\infty} d x e^{i a x}=\frac{i}{a}+\pi \delta(a)
$$

to find

$$
\langle\rho\rangle=-\frac{1}{\pi^{2}} \lim _{t^{\prime} \rightarrow t} \frac{\partial^{2}}{\partial u \partial v}\left[\frac{F(u)+F(v)}{u+v}\right],
$$

where

$$
F(u)=\int_{-\infty}^{\infty} d s \frac{f(s)}{2 s+u}
$$

and the last integral is understood to be a principal value.

Thus given the probability distribution $f(s)$, we need only calculate $F$ (the Hilbert transform of $f$ ), and then evaluate the derivatives and limit in Eq. (41). It is of interest to apply this formalism to the case of a compactly-supported distribution. A simple example is

$$
f(s)=\frac{315}{256 s_{0}^{9}}\left(s-s_{0}\right)^{4}\left(s+s_{0}\right)^{4}, \quad-s_{0} \leq s \leq s_{0},
$$

and $f(s)=0$ for $|s|>s_{0}$. This function is chosen so that $f$ and its first three derivatives are continuous at $s= \pm s_{0}$. Equation (41) now leads to

$$
\begin{aligned}
\langle\rho\rangle= & \frac{1}{512 \pi^{2} s_{0}^{9}}\left\{\left(2205 z^{5}-3150 s_{0}^{2} z^{3}+945 s_{0}^{4} z\right)\left[\ln \left(z+s_{0}\right)-\ln \left|z-s_{0}\right|\right]\right. \\
& \left.-4410 s_{0} z^{4}+4830 s_{0}^{3} z^{2}-672 s_{0}^{5}\right\} .
\end{aligned}
$$

This function is plotted in Fig. 3. Again, we have that at large distances from the mirror

$$
\langle\rho\rangle \sim-\frac{1}{16 \pi^{2} z^{4}}+\cdots, \quad z \gg s_{0} .
$$




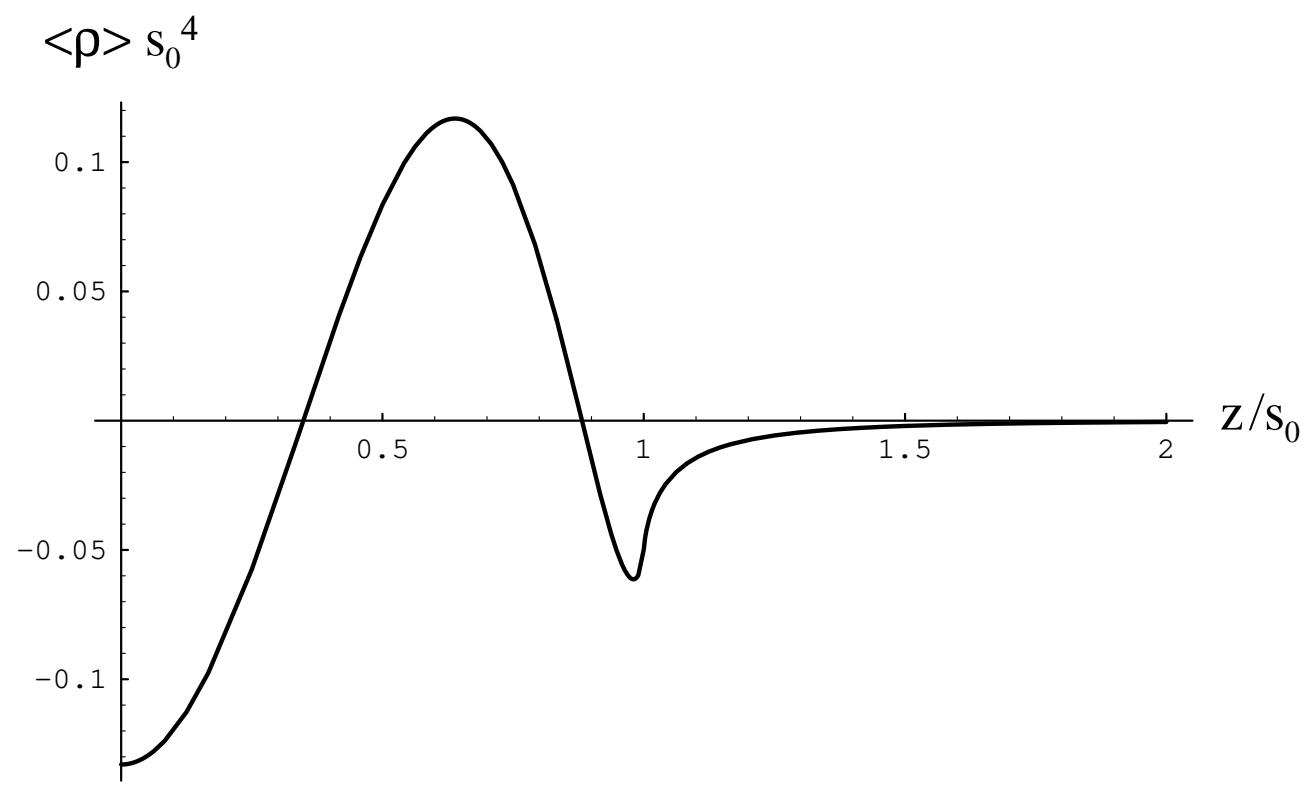

Figure 3:

The mean energy density, $\langle\rho\rangle$, is illustrated for the compact probability distribution, Eq. (43), where $s_{0}$ is the width of the probability distribution. Again, the integral of $\langle\rho\rangle$ over positive $z$ vanishes.

Note that $\langle\rho\rangle$ has a cusp at $z=s_{0}$. If we had chosen a distribution function for which any of the first three derivatives are discontinuous at this point, $\langle\rho\rangle$ would become singular there. Similarly, we could smooth out the cusp by matching more derivatives at this point. We may verify directly that the total energy again vanishes:

$$
\int_{0}^{\infty}\langle\rho\rangle d z=0 .
$$

\section{The Squared Electric Field near a Single Plate}

As noted in the Introduction, the squared electric and magnetic fields diverge in the presence of a perfectly reflecting plate with no position fluctuations. Specifically, one has

$$
\left\langle\mathbf{E}^{2}\right\rangle=-\left\langle\mathbf{B}^{2}\right\rangle=\frac{3}{16 \pi^{2} z^{4}},
$$

so the energy density vanishes:

$$
\langle\rho\rangle=\frac{1}{2}\left(\left\langle\mathbf{E}^{2}\right\rangle+\left\langle\mathbf{B}^{2}\right\rangle\right)=0 .
$$


We may now calculate the squared electric field in the presence of a fluctuating boundary. The two point function for the photon field is

$$
D^{\mu \nu}\left(x, x^{\prime}\right)=\left\langle 0\left|A^{\mu}(x) A^{\nu}\left(x^{\prime}\right)\right| 0\right\rangle .
$$

In the presence of the reflecting plate, this may be expressed as

$$
D^{\mu \nu}\left(x, x^{\prime}\right)=D_{0}^{\mu \nu}\left(x-x^{\prime}\right)+D_{R}^{\mu \nu}\left(x, x^{\prime}\right)
$$

where $D_{0}^{\mu \nu}\left(x-x^{\prime}\right)$ is the two point function in the absence of the boundary, and the renormalized two point function, $D_{R}^{\mu \nu}\left(x, x^{\prime}\right)$, is the correction introduced by the presence of the boundary. In a particular choice of gauge, we have that

$$
D_{0}^{\mu \nu}\left(x-x^{\prime}\right)=\eta^{\mu \nu} G_{0}\left(x-x^{\prime}\right),
$$

and

$$
D_{R}^{\mu \nu}\left(x, x^{\prime}\right)=-\left(\eta^{\mu \nu}+2 n^{\mu} n^{\nu}\right) G_{R}\left(x, x^{\prime}\right) .
$$

Here $G_{0}$ and $G_{R}$ are the scalar two point functions given in Eqs. (7) and (8), respectively, and $n^{\mu}=(0,0,0,1)$ is the unit vector normal to the plate. The renormalized field strength two point function can now be obtained by taking the four dimensional curl in $x$ and in $x^{\prime}$ of Eq. (52). The electric field part of this function is

$$
\left\langle E_{i}(x) E_{j}\left(x^{\prime}\right)\right\rangle=\left\langle F_{0 i}(x) F_{0 j}\left(x^{\prime}\right)\right\rangle=\partial_{0} \partial_{0}^{\prime}\left\langle A_{i}(x) A_{j}\left(x^{\prime}\right)\right\rangle+\partial_{i} \partial_{j}^{\prime}\left\langle A_{0}(x) A_{0}\left(x^{\prime}\right)\right\rangle .
$$

In the presence of boundary fluctuations, the mean squared electric field is now given by

$$
\left\langle\mathbf{E}^{2}\right\rangle=\lim _{\substack{t^{\prime} \rightarrow t \\ \mathbf{x}^{\prime} \rightarrow \mathbf{x}}}\left(\partial_{t} \partial_{t}^{\prime}-\nabla_{\mathbf{x}} \cdot \nabla_{\mathbf{x}^{\prime}}\right)\left\langle G_{R}\left(x, x^{\prime}\right)\right\rangle=\frac{1}{3}\left\langle T_{\mu}^{\mu}\right\rangle
$$

where $T_{\mu}^{\mu}$ is the trace of the minimal scalar field stress tensor. From Eqs. (32) and (35), we find that

$$
\left\langle\mathbf{E}^{2}\right\rangle=-3\langle\rho\rangle .
$$

Thus our explicit results for the scalar field energy density, $\langle\rho\rangle$, Eqs. (28) and (44), also give us $\left\langle\mathbf{E}^{2}\right\rangle$ for the Gaussian and compact probability distributions, respectively.

It is of interest to note that the Casimir-Polder potential between a polarizable particle with a frequency-independent polarizability $\alpha$ and a conducting boundary is given by

$$
V(z)=-\frac{1}{2} \alpha\left\langle\mathbf{E}^{2}\right\rangle .
$$

Thus Figs. 2 and 3 are also plots of $2 V(z) /(3 \alpha)$ for the Gaussian and compact probability distributions, respectively. In both cases, there is a minimum in $V(z)$ at a finite distance from the mean position of the boundary, at which the particle could apparently become trapped. This should probably not be taken too seriously. In the case of Gaussian fluctuations there is a nonzero probability to find the mirror to the right of the minimum of $V(z)$. In the case of the compact probability distribution, Eq. (43), the mirror may be found at any location to the left of the minimum of $V(z)$. 


\section{$5 \quad$ The Energy Density between Two Plates}

Here we will address the problem of finding the mean energy density for a minimally coupled, massless scalar field between a pair of parallel plates. As before, the field is assumed to vanish on the plates, but their positions are allowed to fluctuate. First suppose that the plates are fixed at $z=\eta$ and $z=\sigma$, respectively. The two point function may be constructed as an image sum:

$$
\begin{aligned}
G\left(x, x^{\prime}\right) & =-\frac{1}{4 \pi^{2}}\left[\sum_{m=-\infty}^{\infty} \frac{1}{\Delta \tau^{2}-\left[z-z^{\prime}+2 m(\sigma-\eta)\right]^{2}}\right. \\
& \left.-\sum_{m=-\infty}^{\infty} \frac{1}{\Delta \tau^{2}-\left[z+z^{\prime}+2 m \sigma-2(m+1) \eta\right]^{2}}\right]
\end{aligned}
$$

where $\Delta \tau^{2}=\Delta t^{2}-\Delta \mathbf{x}_{t}^{2}=\Delta t^{2}-\Delta x^{2}-\Delta y^{2}$. The renormalized two point function is obtained by subtracting $G_{0}\left(x, x^{\prime}\right)$, which amounts to omitting the $m=0$ term in the first summation. The result may be written as

$$
\begin{aligned}
G_{R}\left(x, x^{\prime}\right) & =\frac{1}{2(2 \pi)^{3}} \operatorname{Re} \int \frac{d^{3} k}{\omega} e^{i \mathbf{k}_{t} \cdot \Delta \mathbf{x}_{t}} e^{-i \omega \Delta t} \\
\times & {\left[\sum_{m=-\infty}^{\infty} e^{i k_{z}\left[z-z^{\prime}+2 m(\sigma-\eta)\right]}-\sum_{m=-\infty}^{\infty} e^{i k_{z}\left[z+z^{\prime}+2 m \sigma-2(m+1) \eta\right]}\right], }
\end{aligned}
$$

where the prime on a summation denotes that the $m=0$ term is omitted.

We will assume that the positions of both plates are described by the same probability distribution, $f$. We then have that

$$
\begin{aligned}
\left\langle e^{i k_{z}\left[z-z^{\prime}+2 m(\sigma-\eta)\right]}\right\rangle & =\int d \eta f(\eta) \int d \sigma f(\sigma) e^{i k_{z}\left[z-z^{\prime}+2 m(\sigma-\eta)\right]} \\
& =e^{i k_{z}\left[z-z^{\prime}+2 m(\langle\sigma\rangle-\langle\eta\rangle)\right]} \hat{f}^{2}\left(2 m k_{z}\right)
\end{aligned}
$$

and

$$
\left\langle e^{i k_{z}\left[z+z^{\prime}+2 m \sigma-2(m+1) \eta\right]}\right\rangle=e^{i k_{z}\left[z+z^{\prime}+2 m\langle\sigma\rangle-2(m+1)\langle\eta\rangle\right]} \hat{f}\left(2 m k_{z}\right) \hat{f}\left(2(m+1) k_{z}\right) .
$$

The mean energy density is given by Eq. (25). If we combine this expression with the above results, we find

$$
\begin{array}{r}
\langle\rho\rangle=-\frac{1}{8 \pi^{2}} \lim _{\Delta t \rightarrow 0} \operatorname{Re}\left[\frac{\partial^{2}}{\partial t^{2}}\left(\frac{-i}{\Delta t}\right) \int_{-\infty}^{\infty} d k_{z} e^{i\left|k_{z}\right| \Delta t} \sum_{m=-\infty}^{\infty} e^{2 i\left|k_{z}\right| m a} \hat{f}^{2}\left(2 m k_{z}\right)+\right. \\
\left.\left(\frac{1}{4} \frac{\partial^{2}}{\partial z^{2}}-\frac{\partial^{2}}{\partial t^{2}}\right)\left(\frac{-i}{\Delta t}\right) \int_{-\infty}^{\infty} d k_{z} e^{i\left|k_{z}\right| \Delta t} \sum_{m=-\infty}^{\infty} e^{2 i\left|k_{z}\right|(m a+z)} \hat{f}\left(2 m k_{z}\right) \hat{f}\left(2(m+1) k_{z}\right)\right]
\end{array}
$$

where we have set $\langle\sigma\rangle=0$ and $a=\langle\eta\rangle$, so $a$ is the mean separation between the plates. 
We now employ Eq. (40) and the identity [11]

$$
\sum_{m=-\infty}^{\infty} \frac{1}{m-a}=-\pi \cot \pi a
$$

After the derivatives have been evaluated, our final result may be expressed as

$$
\langle\rho\rangle=\langle\rho\rangle_{1}+\langle\rho\rangle_{2}
$$

where

$$
\langle\rho\rangle_{1}=-\frac{\pi^{2}}{1440} \int_{-\infty}^{\infty} d s \int_{-\infty}^{\infty} d r \frac{f(s) f(r)}{(s+r+a)^{4}}
$$

and

$$
\langle\rho\rangle_{2}=-\frac{\pi^{2}}{48} \int_{-\infty}^{\infty} d s \int_{-\infty}^{\infty} d r f(s) f(r) \frac{2 \sin ^{2}\left[\frac{\pi(z+r)}{s+r+a}\right]-3}{(s+r+a)^{4} \sin ^{4}\left[\frac{\pi(z+r)}{s+r+a}\right]} .
$$

Let us first discuss the limit in which the position of both plates is precisely defined. In this case, we take $f(s)=\delta(s)$ and obtain

$$
\langle\rho\rangle_{1}=-\frac{\pi^{2}}{1440 a^{4}}
$$

and

$$
\langle\rho\rangle_{2}=\frac{\pi^{2}}{48} \frac{2 \sin ^{2}\left(\frac{\pi z}{a}\right)-3}{a^{4} \sin ^{4}\left(\frac{\pi z}{a}\right)} .
$$

This is just the usual result [1]; $\langle\rho\rangle_{1}$ is now the energy density for a conformal scalar field, and $\langle\rho\rangle_{2}$ diverges on the boundaries.

Now suppose that we take $f(s)$ to be a function with a finite width, and whose first three derivatives are finite. The integrals in Eqs. (64) and (65) contain poles in the ranges of integration, but the integrals are well-defined as principal value or generalized principal value integrals [12]. That is, we use identities of the form

$$
\int_{-\infty}^{\infty} d s \frac{f(s)}{(s-a)^{4}}=-\frac{1}{3} \int_{-\infty}^{\infty} d s \frac{f^{\prime}(s)}{(s-a)^{3}}=\frac{1}{6} \int_{-\infty}^{\infty} d s \frac{f^{\prime \prime}(s)}{(s-a)^{2}}=-\frac{1}{6} \int_{-\infty}^{\infty} d s \frac{f^{\prime \prime \prime}(s)}{s-a} .
$$

Thus we see that both $\langle\rho\rangle_{1}$ and $\langle\rho\rangle_{2}$ will be finite everywhere.

We may now integrate the finite energy density on $z$ to obtain the mean energy per unit area:

$$
E=\int_{0}^{a}\langle\rho\rangle d z=E_{1}+E_{2}
$$

where

$$
E_{1}=a\langle\rho\rangle_{1}
$$

and

$$
E_{2}=\int_{-\infty}^{\infty} d s \int_{-\infty}^{\infty} d r f(s) f(r) H(s, r)
$$


and $H(s, r)$ is defined by

$$
H(s, r)=-\frac{\pi}{48} \frac{\cos \left[\frac{\pi r}{s+r+a}\right] \sin ^{3}\left[\frac{\pi(z+r)}{s+r+a}\right]-\sin ^{3}\left[\frac{\pi r}{s+r+a}\right] \cos \left[\frac{\pi(z+r)}{s+r+a}\right]}{(s+r+a)^{3} \sin ^{3}\left[\frac{\pi r}{s+r+a}\right] \sin ^{3}\left[\frac{\pi(z+r)}{s+r+a}\right]} .
$$

In order to discuss the case of plates which are highly localized in position, we need to Taylor expand $H(s, r)$ around $s=r=0$ :

$$
H(s, r) \approx-\frac{1}{48 \pi^{2}}\left(\frac{1}{s^{3}}+\frac{1}{r^{3}}\right)+\frac{\pi^{2}(s+r)}{720 a^{4}}+\cdots
$$

Although the leading term in this expansion is singular at $s=0$ or $r=0$, its contribution to $E_{2}$ vanishes because of the symmetry of the probability distribution, $f(s)=f(-s)$. All subsequent terms in the expansion of $H(s, r)$ vanish at $s=r=0$. Thus we find that if we first form the total energy per unit area of the plates, and then take the limit in which $f(s) \rightarrow \delta(s)$, the result is the same as for the conformal scalar field:

$$
E_{1} \rightarrow-\frac{\pi^{2}}{1440 a^{3}}, \quad E_{2} \rightarrow 0 .
$$

In both cases, we now find the same, negative Casimir energy. (Note that, in general, the sign of a Casimir energy is very difficult to predict in advance of an explicit calculation, and can depend upon both boundary conditions and the dimensionality of spacetime [13].)

\section{Summary and Conclusions}

In the previous sections, we have seen that position fluctuations of a reflecting boundary are capable of removing divergences in the renormalized values of local observables, such as $\left\langle\varphi^{2}\right\rangle$ and $\left\langle T_{\mu \nu}\right\rangle$. In the case of the massless, minimally coupled scalar field, such fluctuations also remove the discrepancy between the spatial integral of the renormalized energy density, $\int\left\langle T_{t t}\right\rangle d^{3} x$, and the renormalized expectation value of the Hamiltonian, $\langle H\rangle$. Position fluctuations are necessary if one is to treat the mirror as a quantum mechanical object.

Of course, for real mirrors the mass is likely to be so large that the position uncertainty $\Delta$ is very small. In this case, the cutoff in reflectivity due to dispersion will normally be the dominant effect. Dielectric materials become transparent to electromagnetic radiation at wavelengths shorter than about the plasma wavelength, $\lambda_{p}$. So long as the position uncertainty is small compared to this length, $\Delta \ll$ $\lambda_{p}$, dispersive effects are dominant, and the position fluctuations may be ignored. However, if one could arrange to prepare a mirror in a quantum state in which $\Delta>\lambda_{p}$, the position fluctuation effects discussed in this paper would become dominant.

In the area of gravitational physics, the situation is rather different. Here it is also possible to have horizons which act as boundaries for quantized fields, and for 
the renormalized expectation value of the stress tensor to diverge on the horizon. As discussed in the Introduction, examples include the event horizon of Schwarzschild spacetime in the Boulware vacuum, the Cauchy horizon in Misner space, and possibly the chronology horizon in a spacetime containing closed timelike curves. Now there is no natural cutoff at high frequencies, and in fact higher frequency modes tend to couple more strongly to gravity by virtue of their larger energy. It is of course possible that a more complete quantum theory of gravity will introduce an effective cutoff at the Planck scale. At the present, any discussion of Planck scale physics must be regarded as highly speculative. Nonetheless, position fluctuations of the horizon would seem to provide a possible way to avoid divergent stress tensors. It is plausible that the location of a spacetime horizon undergoes position fluctuations due either to the quantum nature of gravity ("active fluctuations") [14], or to fluctuations of the stress tensor of quantum matter fields ("passive fluctuations") [15]. This is a topic requiring further study.

Acknowledgements: We would like to thank Bruce Jensen and Michael Pfenning for comments on the manuscript. This work was supported in part by the National Science Foundation (Grant No. PHY-9507351) and by Conselho Nacional de Desevolvimento Cientifico e Tecnológico do Brasil (CNPq).

\section{References}

[1] See, for example, S. A. Fulling, Aspects of Quantum Field Theory in Curved Spacetime, (Cambridge University Press, Cambridge, England, 1989), p. 105-106.

[2] D. Deutsch and P. Candelas, Phys. Rev. D 20, 3063 (1979); P. Candelas, Ann. Phys. (N.Y.) 143, 241 (1982).

[3] G. Kennedy, R. Critchley, and S. Dowker, Ann. Phys. (N.Y.) 125, 346 (1980).

[4] N.F. Svaiter and B.F. Svaiter, J. Math. Phys. 32, 175 (1991).

[5] N.F. Svaiter and B.F. Svaiter, J. Phys. A 25, 979 (1992).

[6] W.G. Unruh, Phys. Rev. D 14, 870 (1976); 15, 365 (1977).

[7] M. Visser, Phys. Rev. D 54, 5116 (1996), gr-qc/9604008.

[8] W.A. Hiscock and D.A. Konkowski, Phys. Rev. D 26, 1225 (1982).

[9] L.H. Ford, Phys. Rev. D 51, 1692 (1995).

[10] S.W. Hawking, Phys. Rev. D 46, 603 (1992).

[11] L.B.W. Jolley, Summation of Series, (Dover, New York, 1961), p 100. 
[12] K.T.R. Davies and R.W. Davies, Can. J. Phys. 67, 759 (1989); K.T.R. Davies, R.W. Davies, and G. D. White, J. Math. Phys. 31, 1356 (1990).

[13] See, for example, F. Caruso, N. Pinto-Neto, B.F. Svaiter, and N.F. Svaiter, Phys. Rev. D 43, 1300 (1991).

[14] L.H. Ford and N.F. Svaiter, Phys. Rev. D 56, 2226 (1997), gr-qc//9704050.

[15] R.D. Sorkin, Two Topics concerning Black Holes: Extremality of the Energy, Fractality of the Horizon, gr-qc/9508002; How Wrinkled is the Surface of a Black Hole?, gr-qc/9701056; A. Casher, F. Englert, N. Itzhaki, and R. Parentani, Black Hole Horizon Fluctuations, Nucl. Phys. B484, 419 (1997), hep-th/9606106. Note, however, criticisms of these papers in Ref. [14]. 